\title{
Complicações da blefaroplastia superior
}

\author{
Superior eyelid blepharoplasty and complications
}

Silvana Schellini Artioli ${ }^{1}$, Vanessa Grandi Valezi², Walberto Passos ${ }^{3}$

\begin{abstract}
Resumo
A blefaroplastia tem sido realizada com grande frequência devido à valorização da cirurgia estética. As complicações relacionadas a este procedimento são raras. Este relato de caso tem como objetivo enfatizar um tipo de complicação que pode ocorrer.

T.J.S.P., 45 anos de idade, sexo feminino, branca, procedente de Botucatu, apresentava dermatocálase bilateral. Optou-se pela realização de blefaroplastia superior bilateral, realizada removendo-se pele e gordura da pálpebra superior. No pós-operatório foi observado ptose palpebral à esquerda. Optou-se pela cirurgia para reinserção do músculo levantador da pálpebra superior à esquerda. Um mês após a reinserção, observou-se retração palpebral superior nos dois olhos. A paciente foi, então, submetida à cirurgia para a correção de retração palpebral por desinserção do músculo de Müller. Dois meses após esta última cirurgia, a paciente apresentava ptose palpebral à direita e piora da retração palpebral à esquerda. Nova cirurgia para correção da retração palpebral foi feita, colocando-se enxerto livre de esclera para alongamento do levantador da pálpebra superior esquerda, com bom resultado.

Foi apresentada uma paciente submetida à blefaroplastia e que desenvolveu no pós-operatório quadro de ptose, seguida de retração palpebral. Estes dois quadros podem ocorrer como complicação de blefaroplastia e o cirurgião deve estar atento para manejar de forma apropriada, a fim de obter o resultado que a paciente espera ter.
\end{abstract}

Descritores: Blefaroplastia/efeitos adversos; Cirurgia plástica; Relatos de casos

\begin{abstract}
The blepharoplasty has been performed with great frequency due to the appreciation of aesthetic surgery. The complications related to this procedure are rare. This case report aims to emphasize complications that can occur. T.J.S.P., 45 years old, female, white, resident of Botucatu City, presented dermatocalase bilaterally. We opted to perform upper blepharoplasty bilateral, by removing skin and fat of the upper eyelid. In the postoperative period was observed left eyelid ptosis. We decided to reinsert the levator muscle of the left upper eyelid. One month after the ptosis correction, retraction was observed in both upper eyelid. The patient was then submitted to surgery for the correction of eyelid retraction by disinsertion of the muscle of Muller. Two months after this last surgery, the patient presented right eyelid ptosis and worsening of eyelid retraction to the left. New surgery for correction of the eyelid retraction was made, using free graft sclera for elongation of the left eyelid levator muscle, with good results.

The authors presented a patient who underwent a blepharoplasty and developed ptosis in the post-operative period, followed by eyelid retraction. These two possibilities may occur as a complication of blepharoplasty and the surgeon must be careful to handle in an appropriate way, in order to get the result that the patient expects to have.
\end{abstract}

Keywords: Blepharoplasty/adverse effects; Plastic surgery; Case reports

\footnotetext{
'Universidade Estadual "Júlio de Mesquita Filho" (UNESP) - Botucatu (SP), Brasil;

${ }^{2}$ Oftalmologista - Ourinhos (SP), Brasil;

${ }^{3}$ Oftalmologista - Arapiraca (AL), Brasil.

Trabalho realizado na Faculdade de Medicina de Botucatu, Universidade Estadual "Júlio de Mesquita Filho" (UNESP) - Botucatu (SP), Brasil.
}

Os autores declaram inexistir conflitos de interesse

Recebido para publicação em 29/12/2010 - Aceito para publicação em 26/6/2011 


\section{INTRODUÇÃO}

A

blefaroplastia tem sido realizada com grande frequência devido à valorização da cirurgia estética $^{(1)}$. Suas complicações não são comuns e raramente permanentes, com consequências funcionais ou estéticas ${ }^{(2)}$.

Dentre os problemas que podem surgir após a blefaroplastia, as complicações mais comuns são: ptose da pálpebra superior, lagoftalmia causada pela ressecção incorreta da pele, cicatrizes, anomalias da prega palpebral, retração, ectrópio etc ${ }^{(3-8)}$.

As complicações que envolvem a pálpebra inferior são muito mais marcantes, em especial quando ocorre o ectrópio da pálpebra inferior por ressecção exagerada de pele em indivíduo que muitas vezes já apresenta flacidez dos ligamentos palpebrais.

O objetivo deste relato de caso é enfatizar um tipo raro de complicação que pode ocorrer em blefaroplastias da pálpebra superior.

\section{Relato de caso}

T.J.S.P., 45 anos de idade, sexo feminino, branca, procedente de Botucatu, foi atendida no ambulatório de Plástica Ocular da Faculdade de Medicina de BotucatuUniversidade Estadual Paulista, apresentando queixa de excesso de pele nas pálpebras superiores.

No exame oftalmológico apresentava dermatocálase bilateral, acuidade visual sem correção de 20/30 no olho direito (OD) e 20/20 no olho esquerdo $(\mathrm{OE})$. Na biomicroscopia não foram observadas alterações relevantes. A tonometria de aplanação era de 20 $\mathrm{mmHg}$ no OD e $18 \mathrm{mmHg}$ no OE. A paciente era saudável, apesar de muito magra.

Optou-se pela realização de blefaroplastia superior bilateral, seguindo a técnica empregada no serviço, pela qual remove-se pele em conjunto com o músculo orbicular pré-septal, com abertura do septo orbitário e remoção da gordura orbitária que está aparente nos bolsões medial e mediano. A cirurgia transcorreu sem intercorrências. Durante o pós-operatório foi observado ptose palpebral à esquerda; à direita a pálpebra superior tangenciava o limbo superior, ou seja, havia discreta retração palpebral à direita (Figura 1).

Optou-se pela realização de cirurgia para reinserção do músculo levantador da pálpebra superior à esquerda. Após a reinserção, observou-se retração palpebral superior nos dois olhos.

A paciente foi, então, submetida à cirurgia para a correção da retração palpebral por desinserção do músculo de Müller. Dois meses após esta última cirurgia a paciente apresentava ptose palpebral à direita e retração palpebral à esquerda, agora mais importante que antes da cirurgia anterior (Figura 2). Foi, então, formulada a hipótese de doença de Graves e solicitadas dosagens de T3, T4, TSH e TRAB, cujos resultados foram negativos.

Nova cirurgia para correção da retração palpebral foi feita, colocando-se enxerto livre de esclera para alon-

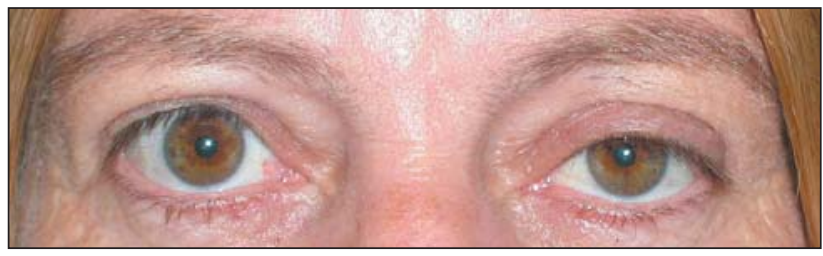

Figura 1: Paciente em pós-operatório, apresentando retração da pálpebra superior do olho direito e ptose na pálpebra superior do olho esquerdo

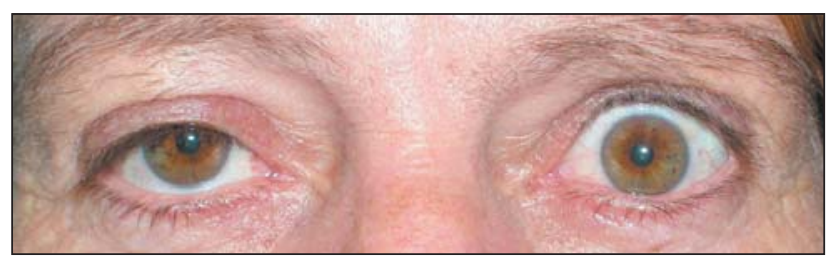

Figura 2: Após correção da ptose à esquerda, ptose à direita e retração palpebral à esquerda
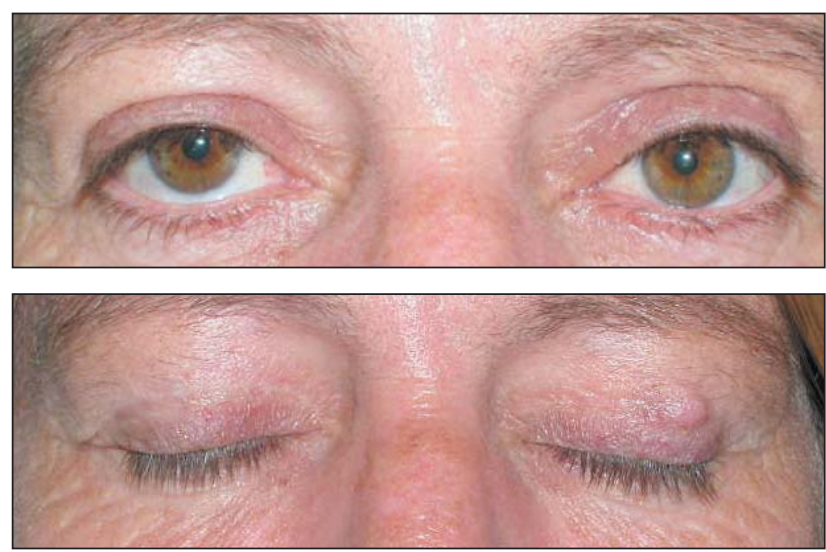

Figura 3: Após a correção da retração da pálpebra superior esquerda com enxerto de esclera, o posicionamento ficou mais adequado (A), mas houve a formação de um granuloma (B)

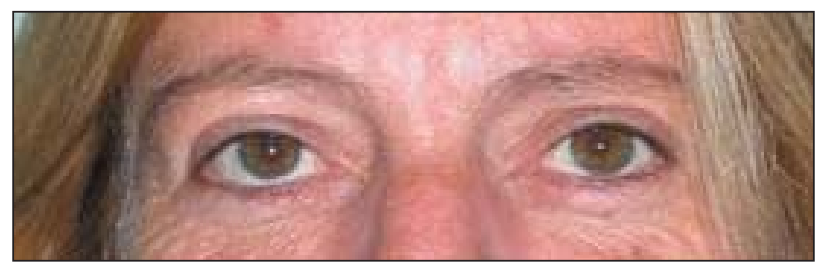

Figura 4: Resultado final

gamento do levantador da pálpebra superior esquerda (Figura 3A). Verificou-se a presença de granuloma na pálpebra superior após esta cirurgia (Figura 3B), o qual foi removido. Cinco meses após a exérese do granuloma foi realizada a reinserção do levantador da pálpebra superior do olho direito, ficando a paciente satisfeita (Figura 4).

Mesmo com os exames de função tireoidina normais, após dois anos a paciente necessitou de tireoidectomia parcial, por ter apresentado um nódulo que era hiperfuncionante. 
A paciente encontra-se bem, com posição das pálpebras estáveis.

\section{DiscussÃo}

A blefaroplastia tem sido frequentemente empregada devido à crescente preocupação com a estética, à disputa pelo mercado de trabalho que exige pessoas de melhor aparência e, também, ao cansaço e redução de campo visual superior ${ }^{(1-2)}$.

Apesar de ser uma cirurgia com poucas dificuldades técnicas, o caso relatado evidenciou duas das complicações mais comuns: a ptose e a retração da pálpebra superior.

A ptose pode ter diversas origens. No período préoperatório, é fundamental que se realize um exame minucioso para afastar a possibilidade de se ter desinserção do músculo levantador prévia a cirurgia. Por outro lado, a desinserção do levantador da pálpebra superior é um risco presente durante a cirurgia, podendo ocorrer por lesão da inserção do mesmo na placa tarsal. No período pós-cirúrgico, a ptose pode ocorrer secundariamente ao edema, hematoma e toxicidade pelo anestésico ${ }^{(9-12)}$.

Por sua vez, a retração palpebral pode decorrer da retirada excessiva de pele, reinserção incorreta do levantador, ou doença de Graves pré-existente ${ }^{(13-15)}$. Devido ao fato de a paciente ser muito magra e da retração palpebral ser um achado muito frequente na orbitopatia de Graves, esta deve ser investigada ${ }^{(16)}$, como foi feito neste caso. Entretanto, os exames afastavam a hipótese de doença tireoidiana, o que veio se confirmar dois anos depois da cirurgia.

Portanto, no presente caso, nos pareceu que a paciente desenvolveu a ptose por desinserção do levantador durante o ato operatório. A retração surgiu de uma hipercorreção da ptose e foi de difícil controle. A Lei de Hering faz com que a pálpebra contralateral também responda, atrapalhando ainda mais a abordagem da paciente.

Outras complicações que devem ser consideradas na cirurgia de blefaroplastia superior são: remoção excessiva ou insuficiente da pele, hemorragia, ceratopatia de exposição, ressecção de glândula lacrimal, remoção excessiva de gordura e descontentamento do paciente. O exame pré-operatório deve passar pela avaliação das expectativas do paciente, da acuidade visual, do fenômeno de Bell, da função do músculo orbicular, do posicionamento e do grau de flacidez das pálpebras, do prolapso da glândula lacrimal e da gordura orbital. O planejamento cirúrgico deve ser muito bem feito, com marcação do fuso de pele a ser retirado na cirurgia, deixando pelo menos $20 \mathrm{~mm}$ para o funcionamento normal da pálpebra.A hemorragia é uma das complicações preveníveis no pré-operatório, sendo importante conhecer se o paciente é portador de doenças (como hipertensão arterial) ou se usa medicações que possam dificultar a coagulação ${ }^{(17)}$. Estas atitudes previnem complicações e protegem os médicos de problemas.

Apesar do número relativamente pequeno de complicações relacionadas à blefaroplastia, estas devem se tornar mais frequentes, à medida que aumenta o número de cirurgias. Embora estas complicações raramente levem a perdas funcionais ou alterações estéticas, as mesmas devem ser sempre lembradas, devendo-se estar atento para não confundir uma complicação com uma alteração prévia.

\section{Conclusão}

Foi apresentada uma paciente submetida à blefaroplastia e que desenvolveu no pós-operatório quadro de ptose seguida de retração palpebral. Estes dois quadros podem ocorrer como complicação de cirurgia de blefaroplastia e o cirurgião deve estar atento para manejar de forma apropriada a fim de obter o resultado que a paciente espera ter.

\section{RefERÊNCIAS}

1. Soares EJC, Moura EM, Gonçalves JOR. Cirurgia plástica ocular. São Paulo: Roca; 1997. p. 243-59.

2. Morax S. Complications des blépharoplasties. J Fr Ophtalmol. 2004;27(6 Pt 1):658-74

3. DeMere M, Wood T, Austin W. Eye complications with blepharoplasty or other eyelid surgery. A national survey. Plast Reconstruct Surg. 1974;53(6):634-7.

4. Harley RD, Nelson LB, Flanagan JC, Calhoun JH. Ocular motility disturbances following cosmetic blepharoplasty. Arch Ophthalmol. 1986;104(4):542-4.

5. Hayworth RS, Lisman RD, Muchnick RS, Smith B. Diplopia following blepharoplasty. Ann Ophthalmol. 1984;16(5):448-51.

6. Rainin EA, Carlson BM. Postoperative diplopia and ptosis. A clinical hypothesis based on the myotoxicity of local anesthetics. Arch Ophthalmol. 1985;103(9):1337-9.

7. Graham WP 3rd, Messner KH, Miller SH. Keratoconjunctivitis sicca symptoms appearing after blepharoplasty. The "dry eye" syndrome. Plast Reconstruct Surg. 1976;57(1):57-61.

8. Wiggs EO. Blepharoplasty complications. Trans Sect Ophthalmol Am Acad Ophthalmol Otolaryngol. 1976;81(4 Pt 1):OP603-6.

9. Hornblass A. Ptosis and pseudoptosis and blepharoplasty. Clin Plast Surg. 1981;8(4):811-30.

10. Leone CR Jr. Management of the blepharoplasty patient with ptosis. Ophthalmic Surg. 1988;19(7):515-22.

11. Patipa M, Wilkins RB. Acquired ptosis in patients undergoing upper eyelid blepharoplasty. Ann Ophthalmol. 1984;16(3):266, 268-70.

12. Baylis HI, Sutcliffe T, Fett DR. Levator injury during blepharoplasty. Arch Ophthalmol.1984;102(4):570-1.

13. Adams BJ, Feurstein SS. Complications of blepharoplasty. Ear Nose Throat J. 1986;65(1):6-18.

14. Castañares S. Complications in blepharoplasty. Clin Plast Surg. 1978;5(1):139-65.

15. Lisman RD, Hyde K, Smith B. Complications of blepharoplasty. Clin Plast Surg. 1988;15(2):309-35.

16. Gladstone GJ. Ophthalmologic aspects of thyroid-related orbitopathy. Endocrinol Metab Clin North Am. 1998;27(1):91-100.

17. Dortzbach RK. Ophthalmic plastic surgery: prevention and management of complications. New York: Raven Press; 1994. p. 91-111.

\section{Autor correspondente:}

Silvana Artioli Schellini

Depto. Oftalmologia/ORL/CCP

Faculdade de Medicina de Botucatu (UNESP)

CEP 18618-970 - Botucatu (SP), Brasil

Tel: (14) 3811-6256

e-mail: sartioli@fmb.unesp.br. 